\title{
Scanning microwave-induced thermoacoustic tomography: Signal, resolution, and contrast
}

\author{
Geng Ku and Lihong V. Wang ${ }^{\mathrm{a})}$ \\ Optical Imaging Laboratory, Biomedical Engineering Program, Texas A\&M University, 3120 TAMU, \\ College Station, Texas 77843-3120
}

(Received 12 July 2000; accepted for publication 25 October 2000)

\begin{abstract}
Scanning thermoacoustic tomography was explored in the microwave region of the electromagnetic spectrum. Short microwave pulses were used to induce acoustic waves by thermoelastic expansion in biological tissues. Cross sections of tissue samples were imaged by a linear scan of the samples while a focused ultrasonic transducer detected the time-resolved thermoacoustic signals. Based on the microwave-absorption properties of normal and cancerous breast tissues, the piezoelectric signals in response to the thermoacoustic contrast were investigated over a wide range of electromagnetic frequencies and depths of tumor locations. The axial resolution is related to the temporal profile of the microwave pulses and to the impulse response of the ultrasonic transducer. The lateral resolution is related to the numerical aperture of the ultrasonic transducer as well as to the frequency spectra of the piezoelectric signals in the time window corresponding to the axial resolution. Gain compensation, counteracting the microwave attenuation, was applied to enhance the image contrast. () 2001 American Association of Physicists in Medicine. [DOI: 10.1118/1.1333409]
\end{abstract}

Key words: thermoacoustics, microwave, ultrasonics, tomography, photoacoustics

\section{INTRODUCTION}

Purely microwave imaging of biological tissues has been investigated for a number of years. ${ }^{1-5}$ The advantages of microwave imaging include the use of nonionizing radiation and its relatively good imaging contrast. However, purely microwave imaging has had difficulties in multichannel detection of microwaves without cross coupling, in reconstruction algorithms, and especially in achieving good spatial resolution because of the strong diffraction of microwaves determined by the long wavelength. Purely ultrasound imaging (ultrasonography), an established medical imaging modality, can yield good spatial resolution but has poor contrast for early-stage tumors. Microwave-induced thermoacoustics can potentially bridge the gap and fuse the advantages of the two imaging modalities.

In microwave-induced thermoacoustics, microwave pulses generate acoustic waves in electromagnetically lossy media. This phenomenon was known as the microwaveauditory or microwave-hearing effect in the early years. ${ }^{6}$ Microwave-induced thermoacoustics was used to quantify physical parameters in media such as the power density and the concentration of a given substance. ${ }^{7-9}$ Several investigators employed microwave-induced thermoacoustics in the 1980s for imaging of biological tissues. ${ }^{10-15}$ However, these early works did not produce any tomographic or depthresolved images. Recently, images of biological tissues were computationally reconstructed based on microwave-induced thermoacoustics. ${ }^{16,17}$ This approach requires measurements of a large amount of data around the tissue and postprocessing computation. Our group explored scanning microwave-induced thermoacoustic tomography. ${ }^{18,19}$ Our approach is similar to the conventional ultrasonic B scan except that the ultrasound is produced internally inside the tissue by microwave pulses. This scanning approach can potentially provide real-time imaging and coregistration with ultrasonic B-scan images.

Microwave-induced thermoacoustic imaging is based on the detection of the thermoacoustic signals generated by microwave radiation in the samples. Pulses of microwaves are used to irradiate the samples. Absorbed microwave energy causes thermoelastic expansion, which radiates acoustic waves, termed thermoacoustic waves. An ultrasonic transducer detects the time-resolved thermoacoustic signals. If optical radiation instead of microwave radiation is used, this thermoacoustic phenomenon is better known as photoacoustics. Microwave-induced thermoacoustic imaging shares similar principles with its optical counterpart. ${ }^{20-24}$ However, microwave-induced thermoacoustic imaging may find unique applications in medical imaging because microwave radiation provides a deeper penetration depth in biological tissues than does optical radiation and has different contrast mechanisms.

We further investigated scanning microwave-induced thermoacoustic tomography toward biomedical applications. The piezoelectric signal, which is related to the thermoacoustic contrast, was simulated over a wide range of electromagnetic frequencies and depths of tumor locations based on the microwave-absorption properties of normal and cancerous breast tissues. The imaging resolutions were investigated in relation to the experimental parameters. Images of thick biological tissue samples were acquired experimentally, which clearly revealed both artificially buried tissue objects and intrinsic structures as well. Gain compensation based on electromagnetic attenuation was applied to enhance the image contrast. 


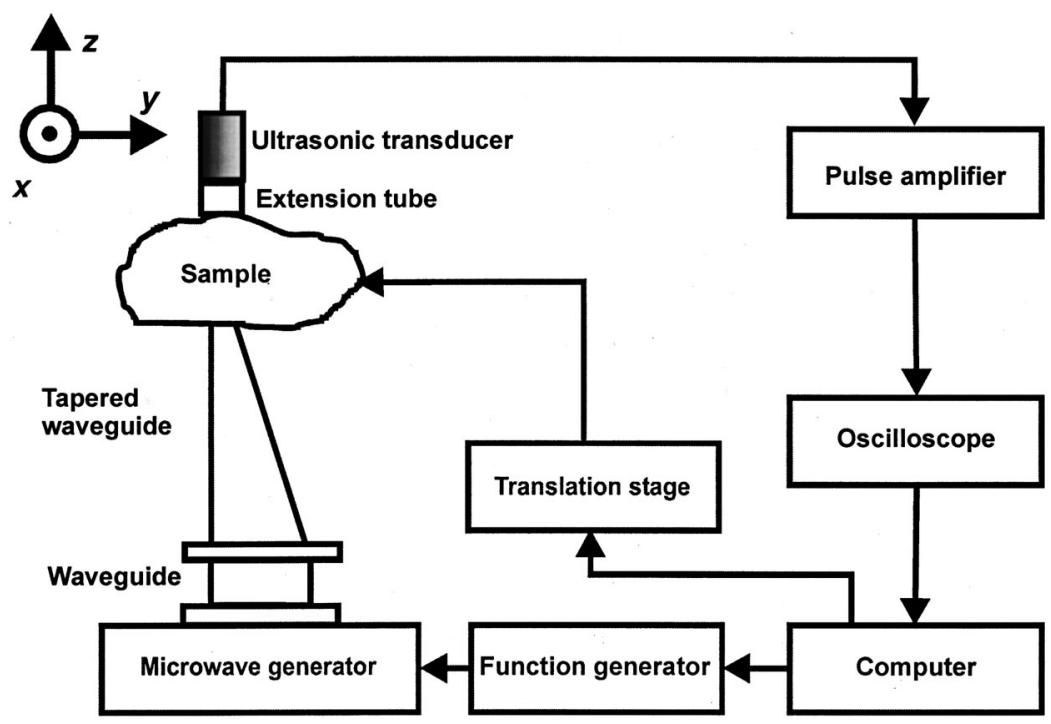

FIG. 1. Experimental setup for scanning thermoacoustic tomography.

\section{METHODS}

The experimental setup for this study is shown in Fig. 1. A Cartesian coordinate system was set up for reference. The $z$ axis was along the acoustic axis pointing upward. The $x$ axis was perpendicular to the drawing plane and pointed outward. The $y$ axis was in the drawing plane pointing to the right. A 3-GHz microwave generator transmitted microwave pulses toward the tissue samples. The pulse width was set to $0.5 \mu$ s for most of the experiments unless it is stated otherwise. The energy of a single microwave pulse was estimated to be $5 \mathrm{~mJ}$, causing a temperature rise of $<1 \mathrm{mK}$ in the tissue samples. A function generator (DS345, Stanford Research System) was employed to trigger the microwave generator, to control the pulse repetition rate of microwave radiation, and to synchronize the sampling by the oscilloscope. Microwave energy was delivered through two waveguides in tandem: a standard rectangular waveguide with a cross section of $72 \mathrm{~mm} \times 34 \mathrm{~mm}$ and a tapered waveguide that narrowed the output cross section to $72 \mathrm{~mm} \times 5 \mathrm{~mm}$. The narrow output port helped concentrate the microwave energy into the imaging zone in the samples near the port; however, the imaging zone far from the port may receive less energy than it would otherwise from the original port as a result of increased diffraction. The object to be imaged was placed on a plastic holder, which was fixed onto a two-dimensional $x-y$ translation stage (MD2, Arrick Robotics). A personal computer controlled the two stepper motors to drive the translation stage in the $x$ and the $y$ directions. An extension tube filled with acoustic-coupling water was mounted onto the ultrasonic transducer. The length of the extension tube was determined such that its focal zone was inside the region of imaging interest in the samples. The bottom surface of the extension tube was in contact with the samples for good acoustic coupling. The central frequency of the ultrasonic transducer (V314, Panametrics) was $1 \mathrm{MHz}$, the bandwidth was $0.6 \mathrm{MHz}$, the diameter was $1.9 \mathrm{~cm}$, the focal length was $2.5 \mathrm{~cm}$, and the focal diameter at $1 \mathrm{MHz}$ was $0.2 \mathrm{~cm}$. The piezoelectric output of the ultrasonic transducer was con- nected to a pulse amplifier. The amplified signal was averaged 10-100 times by an oscilloscope (TDS-640A, Tektronix) and then transferred to the personal computer.

Because the propagation speed of electromagnetic waves is much greater than the speed of sound, the microwave pulses stimulated the entire tissue sample essentially simultaneously. The induced thermoacoustic waves took different amounts of time to reach the ultrasonic transducer. The ultrasonic transducer measured the time of arrival of the thermoacoustic waves. The distance between the acoustic sources and the transducer was calculated by multiplying the time of arrival with the speed of sound in the medium, assuming the speed of sound is constant and known. Because the ultrasonic transducer was focused, it responded primarily to the acoustic sources along its focal column. Consequently, a time-domain signal can be converted into a onedimensional image along the acoustic axis ( $z$ axis), which is similar to an ultrasonic A scan. Scanning the sample along the $x$ or the $y$ axis yielded a two-dimensional cross-sectional image of the sample.

The narrow elongated output port of the tapered waveguide may be considered as a "line source" of electromagnetic radiation. Cylindrical wave propagation was assumed throughout the range of frequencies in our simulation of the thermoacoustic signals, although waveguides are rarely employed in practice for the low-frequency region of the considered electromagnetic spectrum. An electromagnetic wave was emitted from the tapered waveguide and attenuated in tissue approximately as

$$
I(z)=I_{0} \frac{\exp (-2 \alpha z)}{\sqrt{z}}
$$

where $I_{0}$ is the intensity at the output port of the tapered waveguide, $z$ is the distance from the output port to the point of observation along the vertical axis, $I(z)$ is the intensity at $z$, and $\alpha$ is the field-absorption coefficient in the tissue samples. 
The absorption coefficient can be expressed as

$$
\alpha=\omega \sqrt{\frac{\mu \epsilon}{2}\left[\sqrt{1+\left(\frac{\sigma}{\omega \epsilon}\right)^{2}}-1\right]},
$$

where $\omega$ is the angular frequency, $\mu$ is the permeability, $\epsilon$ is the permittivity, and $\sigma$ is the conductivity. In the frequency range of $0.1-10 \mathrm{GHz}$, the dielectric constant (ratio of the permittivity in the material to that in vacuum) has a value of 5-70 for soft tissues, and the conductivity has a value of $0.02-3 \Omega^{-1} \mathrm{~m}^{-1} .^{25-27}$ The complex dielectric properties of tissues at various microwave frequencies determine the propagation and absorption distribution of microwaves. Consequently, the induced thermoacoustic pressure depends on the microwave intensity and the complex dielectric constant of the tissue samples.

A model was used to estimate the microwave-induced thermoacoustic signals. A small breast tumor was embedded in normal breast tissue. The normal breast tissue predominantly determined the microwave attenuation. The thermoacoustic pressure $p$ was proportional to the local absorbed microwave power:

$$
p \propto I_{0} \frac{2 \alpha \exp (-2 \alpha z)}{\sqrt{z}} .
$$

The ultrasonic transducer responded to the components of the thermoacoustic pressure within its response bandwidth and rejected the components outside the bandwidth. The piezoelectric signal $V_{p}$ from the ultrasonic transducer was proportional to the thermoacoustic contrast:

$$
V_{p} \propto\left(p_{t}-p_{n}\right),
$$

where $p_{t}$ and $p_{n}$ were, respectively, the thermoacoustic pressures in the tumor and the normal background tissue at depth $z$, where the tumor and the normal tissue interfaced. Based on Eqs. (3) and (4), we obtained

$$
V_{p} \propto I_{0} \frac{2\left(\alpha_{t}-\alpha_{n}\right) \exp \left(-2 \alpha_{n} z\right)}{\sqrt{z}},
$$

where $\alpha_{t}$ and $\alpha_{n}$ represented the microwave-absorption coefficients of the tumor and the normal breast tissue, respectively.

\section{RESULTS AND DISCUSSION}

Figure 2(a) shows the penetration depth of electromagnetic waves in various human tissues as a function of the electromagnetic frequency in the radio frequency (rf) region, where the penetration depth is the inverse of the absorption coefficient. The penetration depths of muscle and fat tissues are plotted in solid lines. ${ }^{28}$ At the frequency of our experimental setup, $3 \mathrm{GHz}$, the penetration depths for fat and muscle are 9 and $1.2 \mathrm{~cm}$, respectively. The penetration depths of normal and malignant human breast tissues are plotted in dashed lines. ${ }^{29}$ At $3 \mathrm{GHz}$, the penetration depths for malignant and normal breast tissue are approximately 2.3
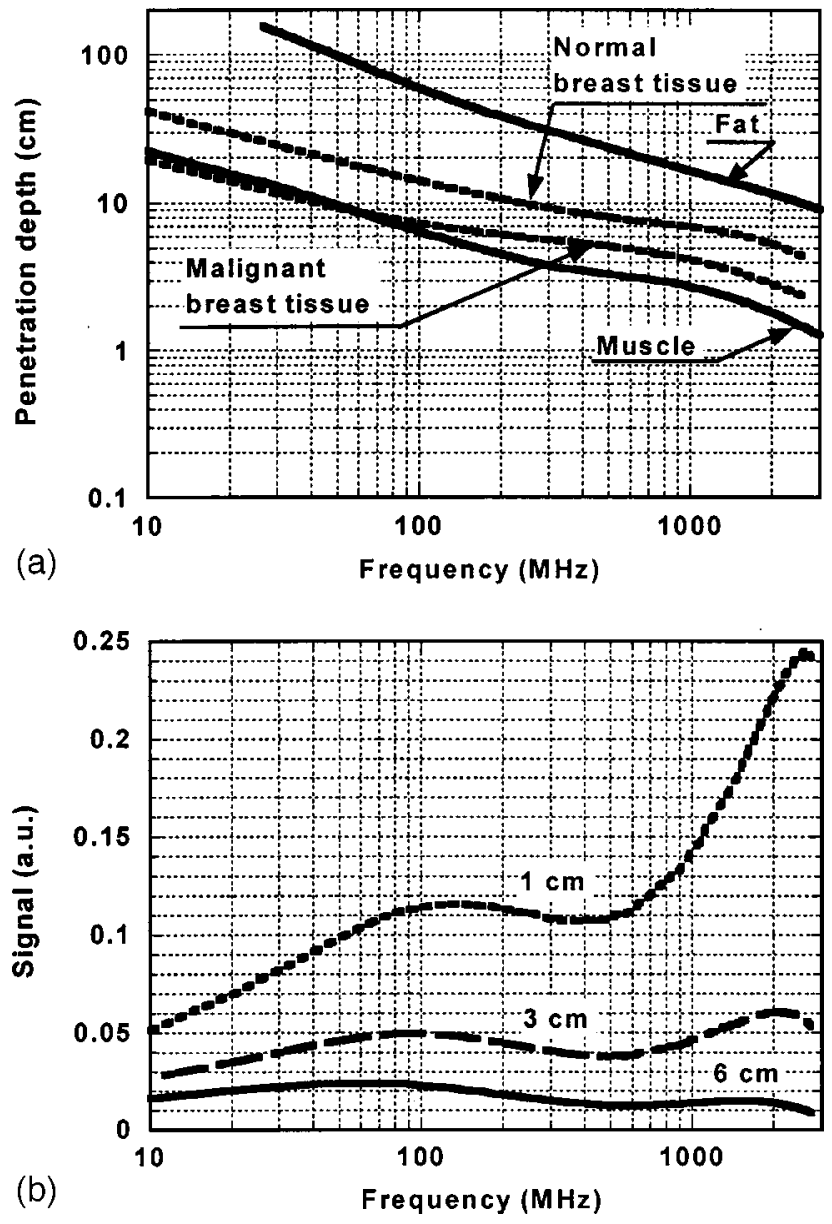

FIG. 2. (a) Penetration depths of various biological tissues versus the microwave frequency; (b) simulated piezoelectric signal in response to a thermoacoustic wave from a tissue sample containing a buried tumor versus the microwave frequency.

and $4.4 \mathrm{~cm}$, respectively. Malignant breast tissues are more strongly absorbing than surrounding normal breast tissues. $^{30,31}$

Water and ion concentrations are key factors in microwave absorption. ${ }^{32}$ Muscle and fat tissues have very high and very low water contents, respectively, and therefore represent the extreme microwave absorption properties. Most other soft tissues have an absorption coefficient in between those for muscle and fat tissues. This wide range of values among various tissues is desired to achieve a high image contrast.

We calculated the piezoelectric signal as a function of the electromagnetic frequency and tumor location using Eq. (5) [Fig. 2(b)]. If the detection system is instrument-noise limited, the signal-to-noise ratio (SNR) of the system is different from this piezoelectric signal by a constant factor. The noise in our detection system was mainly from the pulse amplifier, which was used to amplify the piezoelectric signal from the ultrasonic transducer. The noise remained almost constant in our experiment and was independent of the microwave frequency or the depth of the tumor. The SNR decreased as the tumor location increased because of the increasingly attenuated microwave intensity. When the tumor was located near 
the tissue surface, e.g., at $1 \mathrm{~cm}$ depth, the SNR was better at higher frequencies. When the tumor was located more deeply, the choice of frequency for an optimal SNR was rather broad. This was because the decrease in thermoacoustic pressure was compensated by the increasing difference of the rf-absorption coefficients between the tumor and the normal breast tissues as the frequency increased.

The axial resolution is limited by two factors: the temporal width of the microwave pulse and the temporal width of the impulse response of the ultrasonic transducer. The temporal width of the impulse response of the ultrasonic transducer is inversely proportional to the bandwidth of the ultrasonic transducer. To illustrate the effect of the microwavepulse width on the axial resolution, we simulated the thermoacoustic pressures and the corresponding piezoelectric signals from a microwave-absorbing slab of $5 \mathrm{~mm}$ in thickness using Eq. (3) and the convolution method that was described previously. ${ }^{19}$ The excitation microwave pulses had the same peak power but different pulse widths: $1.0,0.5$, and $0.1 \mu$ s (Fig. 3). There were two dipolar structures corresponding to the two boundaries of the slab. The width of the dipolar structures was determined by the width of the microwave pulses and the impulse response time of the ultrasonic transducer. The distance between the dipolar structures was determined by the acoustic-transit time across the slab. For the 5-mm thick slab, the acoustic-transit time was $3.3 \mu \mathrm{s}$ based on the speed of sound of $\sim 1.5 \mathrm{~mm} / \mu \mathrm{s}$. Pumping with a narrower microwave pulse decreased the width of the dipolar structures and therefore improved the axial resolution because the dipolar structures defined the time window for the axial resolution. The narrower microwave pulses of the same peak pressure also produced smaller signals. Likewise, an ultrasonic transducer of a higher central frequency and a broader bandwidth produced narrower dipolar structures and therefore improved the axial resolution at the expense of signal strength.

The lateral resolution at the focal plane was limited by the focal diameter of the ultrasonic transducer. Based on the ultrasound reciprocity, the focal diameter determined both the beam diameter when the ultrasonic transducer transmits ultrasound and the detection directivity factor when the ultrasonic transducer detects ultrasound. The focal diameter was determined approximately by

$$
\phi_{f}=\lambda_{a} / \mathrm{NA}=c_{a} /\left(\mathrm{NA} f_{a}\right),
$$

where $\lambda_{a}$ was the acoustic wavelength, NA was the numerical aperture of the ultrasonic transducer, $c_{a}$ was the speed of sound, and $f_{a}$ was the central frequency of the piezoelectric signal. The numerical aperture NA was solely determined by the geometric properties of the ultrasonic transducer. The speed of sound $c_{a}$ was relatively constant throughout the frequency range. The central frequency of the piezoelectric signal $f_{a}$ was determined by the frequency spectrum of the thermoacoustic signal in the dipolar structures and by the frequency response of the ultrasonic transducer. Therefore, the lateral resolution was not only related to the ultrasonic parameters, including the numerical aperture and the frequency response of the ultrasonic transducer, but also related
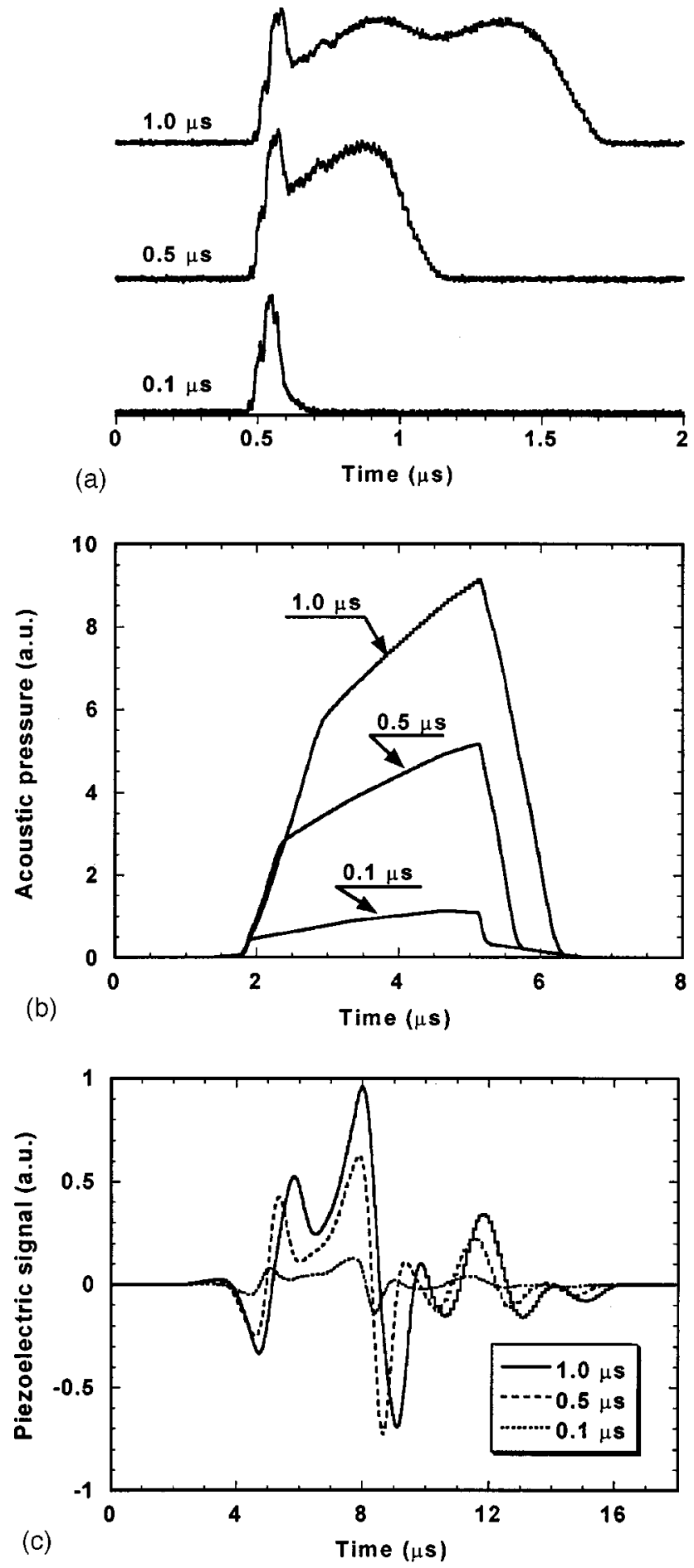

FIG. 3. (a) Waveforms of the microwave pulses of various pulse widths that were tested in the experiment; (b) simulated thermoacoustic waves from a 5-mm-thick microwave-absorbing slab by the microwave pulses; (c) simulated piezoelectric signals of the thermoacoustic waves in (b).

to the frequency content of the thermoacoustic signal in the dipolar structures. A high frequency was obviously desired for high-resolution imaging.

The ultrasonic transducer functioned as a frequency filter to the thermoacoustic signals. The homogeneous tissue between interfaces produced slowly varying thermoacoustic signals. The slowly varying signals were outside the band- 


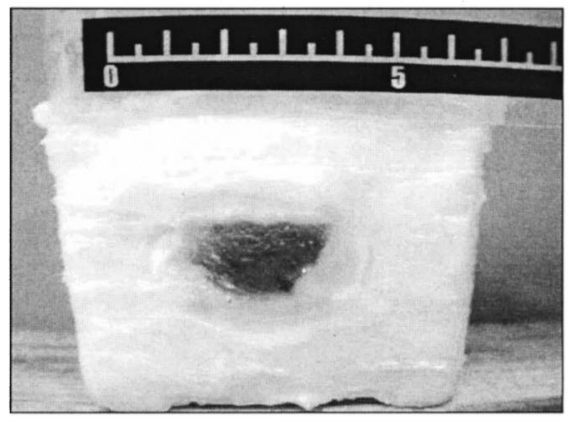

(a)

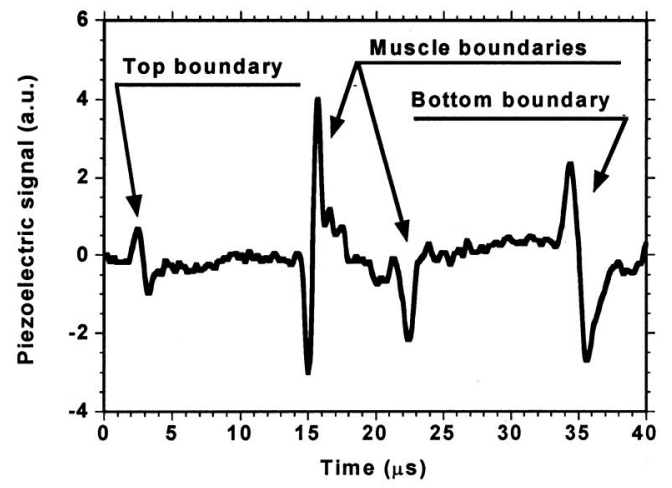

(c)

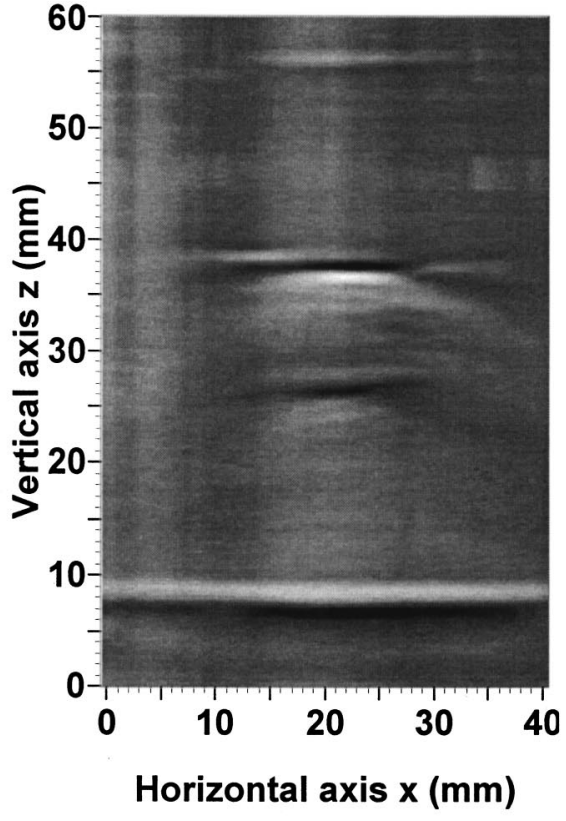

FIG. 4. (a) Photograph of a $y-z$ cross section of a lard sample containing a piece of muscle tissue, which was taken after the sample was imaged with the scanning thermoacoustic imaging system; (b) two-dimensional thermoacoustic image of the cross section; (c) typical temporal thermoacoustic signal corresponding to a vertical line in the two-dimensional thermoacoustic image.

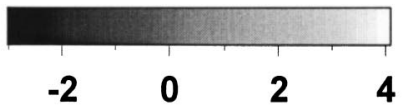

Piezoelectric signal (a.u.)

(b)

width of the ultrasonic transducer and therefore were rejected. The variations in microwave absorption at tissue interfaces caused abrupt changes in the thermoacoustic pressure. The abruptly varying signals had frequency components falling into the response bandwidth of the ultrasonic transducer and made a primary contribution to the piezoelectric signals of the ultrasonic transducer. An ultrasonic transducer of a higher central frequency would provide better lateral resolution. Such a transducer usually has a broader bandwidth and would consequently yield better axial resolution as well. If the resolution were improved by varying the ultrasonic parameters, the SNR would be reduced because the volume of the thermoacoustic signal contributing to the piezoelectric signal is reduced. Therefore, there is a tradeoff between imaging resolution and SNR.

Two samples were imaged with our microwave-induced thermoacoustic imaging system. The background of the first sample was made intentionally as homogeneous as possible. A piece of muscle tissue was embedded into melted lard before it solidified, where the lard was used for its homogeneity. The sample was cut across to reveal the cross section after it was imaged with scanning thermoacoustic tomography (Fig. 4). The time-resolved thermoacoustic signals were acquired at each step while the sample was scanned horizontally along the $y$ axis with a step size of $1 \mathrm{~mm}$. Each vertical line in this two-dimensional thermoacoustic image was obtained from a temporal thermoacoustic waveform. Figure 3 (c) shows the temporal thermoacoustic signal for $y$ equal to $23 \mathrm{~mm}$ as an example. The earliest arriving signal came from the upper surface of the lard. The following signals corresponded to the two surfaces of the muscle and the bottom surface of the lard.

Because the sample was in the near field of microwave, a logical question was whether the heterogeneity of the electromagnetic field would cause heterogeneity in thermoacoustic images. As shown in Fig. 4, the signal from the background lard is very weak, indicating the heterogeneity of the electromagnetic field does not affect thermoacoustic imaging significantly. The heterogeneity of the electromagnetic field is of the scale of the wavelength, several centimeters in biological tissue at the 3-GHz frequency. Spatial variations of this scale correspond to low-frequency thermoacoustic signals and are therefore filtered out by the ultrasonic transducer.

In the second sample the background was left with heterogeneity. A piece of swine muscle tissue of $\sim 5 \mathrm{~mm}$ in thickness was buried inside a piece of swine fat tissue. The fat tissue was naturally separated into several layers by thin $(<1 \mathrm{~mm}$ in thickness) connective tissue that has greater microwave absorption than the adjacent fat tissue. A cross section of the sample was exposed and photographed after the sample was imaged with our scanning thermoacoustic imaging setup (Fig. 5). Both the buried muscle and the connective tissue are clearly visible in the thermoacoustic image.

Because of microwave attenuation, the deeper structures in the tissue samples received less microwave radiation and produced weaker thermoacoustic signals than did the shallower structures. Consequently, the deeper structures were 


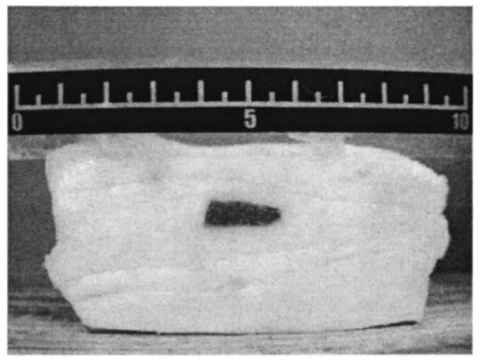

(a)

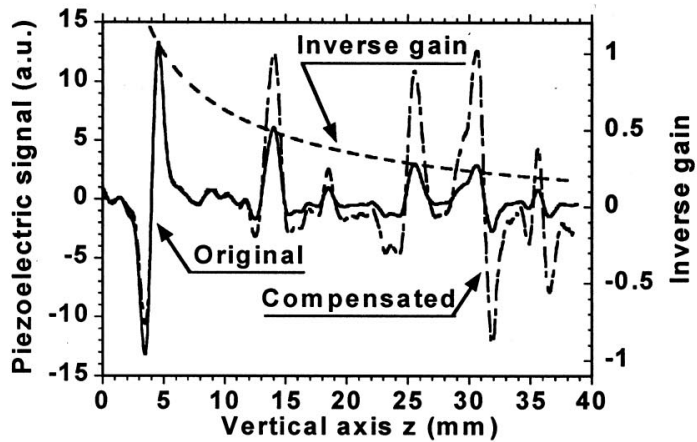

(c)

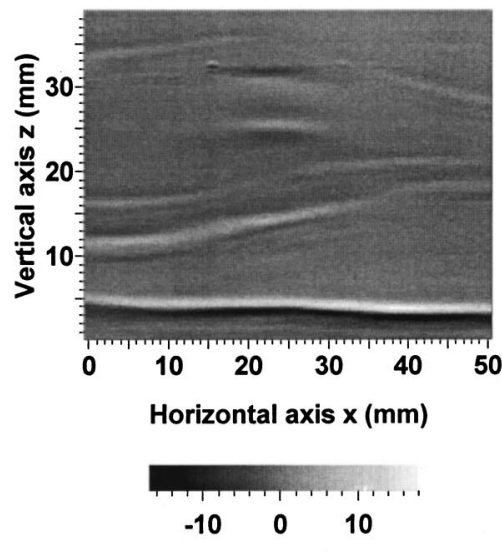

Piezoelectric signal (a.u.)

(b)

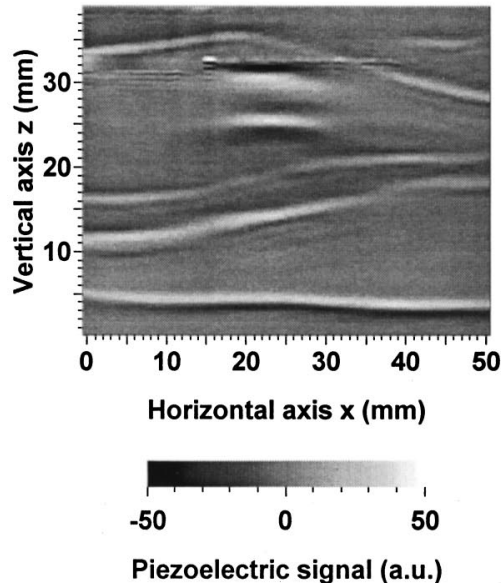

(d)
FIG. 5. (a) Photograph of a $y-z$ cross section of a fat-tissue sample containing a piece of muscle tissue, which was taken after the sample was imaged with the scanning thermoacoustic imaging system; (b) two-dimensional thermoacoustic image of the cross section; (c) gain compensation to a typical piezoelectric signal; (d) twodimensional thermoacoustic image of the cross section after the gain compensation. not imaged as clearly as the shallower ones. To enhance the image contrast, we compensated the piezoelectric signal with the following gain factor:

$$
g(z)=\sqrt{z} \exp (2 \alpha z),
$$

which is the inverse of microwave intensity attenuation. In Fig. 5(c), the original piezoelectric signal for $y$ equal to 20 $\mathrm{mm}$ was plotted with a solid line. The microwave-induced thermoacoustic signal decreased for increasing distance $z$. The inverse of the gain factor was shown in the figure with a dashed line for a comparison with the decay of the original piezoelectric signal. The deeper signals were compensated with greater gains. The compensated data, shown as a pointdashed line, had a nearly constant envelope throughout the imaged depth. The gain-compensated image [Fig. 5(d)] shows the deeper structures clearly as well. Some interference from the pulse amplifier was also amplified by the "'gain compensation," shown as the artifacts in the image near the upper surface of the muscle tissue. This technique is similar to the "time-gain compensation" in conventional ultrasonography. Of course, the decay constant in Eq. (7) depends on the types of tissue being imaged, but it can be adjusted dynamically to optimize the image quality.

It is worth noting that the tissue samples also function as a frequency filter to the thermoacoustic signals. Higherfrequency components experience greater attenuation than the lower-frequency ones. This attenuation is severe for the high-frequency components that pass through a long path in the tissue samples. The preferential reduction in the highfrequency components would adversely affect the imaging resolution.

We also assumed that the tissue samples were electromagnetically heterogeneous and acoustically homogeneous, and hence we revealed the electromagnetic contrast. However, if the tissue samples were also acoustically heterogeneous, additional measures would have to be taken to account for the effect of the acoustic heterogeneity. One straightforward method would be to co-register the thermoacoustic images with conventional ultrasonograms of the same imaging planes. Of course, the purpose of thermoacoustic tomography is to image objects that do not have acoustic contrast and cannot be imaged by conventional ultrasonography.

\section{CONCLUSIONS}

We have investigated scanning microwave-induced thermoacoustic tomography both experimentally and theoretically. Our theoretical analysis of the SNR showed that for malignant tumors located at a $3-6 \mathrm{~cm}$ depth, the choice of the optimal microwave frequency was broad, ranging between $100 \mathrm{MHz}$ and $3 \mathrm{GHz}$. The axial resolution was obtained by measuring the time-resolved microwave-induced thermoacoustic signals. Depth-resolved tomographic images were acquired directly without resorting to computational 
image reconstruction-inverse problems. The key factors affecting the axial resolution include the pulse width of microwave radiation and the bandwidth of the ultrasonic transducer. The lateral resolution was achieved by the use of a focused ultrasonic transducer. The factors affecting the lateral resolution include the numerical aperture of the ultrasonic transducer and the central frequency of the piezoelectric signal in response to the thermoacoustic waves. The central frequency of the piezoelectric signal was determined by the frequency response of the ultrasonic transducer and the frequency spectrum of the microwave-induced thermoacoustic wave in the resolvable dipolar structures. Gain compensation can be used to counteract the microwave attenuation and improve the image contrast.

\section{ACKNOWLEDGMENTS}

We thank Dr. K. Chang for a helpful discussion. This project was sponsored in part by National Institutes of Health Grants No. R29 CA68562, No. R01 CA71980, and No. R21 CA83760, National Science Foudation Grant No. BES9734491, and Texas Higher Education Coordinating Board Grant No. ARP 000512-0123-1999.

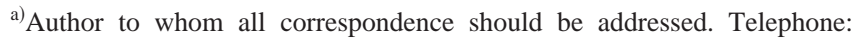
979-847-9040; fax: 979-845-4450; electronic mail: LWang@tamu.edu URL: http://oilab.tamu.edu.

${ }^{1}$ L. E. Larsen and J. H. Jacobi, in Medical Applications of Microwave Imaging (IEEE, Piscataway, NJ, 1986).

${ }^{2}$ J. C. Lin, "Frequency optimization for microwave imaging of biological tissues," Proc. IEEE 73, 374-375 (1985).

${ }^{3}$ S. Caorsi, A. Frattoni, G. L. Gragnani, E. Nortino, and M. Pastorino, "Numerical algorithm for dielectric-permittivity microwave imaging of inhomogeneous biological bodies," Med. Biol. Eng. Comput. 29, NS37-44 (1991).

${ }^{4}$ M. S. Hawley, A. Broquetas, L. Jofre, J. C. Bolomey, and G. Gaboriaud, "Microwave imaging of tissue blood content changes," J. Biomed. Eng. 13, 197-202 (1991).

${ }^{5}$ P. M. Meaney, K. D. Paulsen, and J. T. Chang, "Near-field microwave imaging of biologically-based materials using a monopole transceiver system," IEEE Trans. Microwave Theory Tech. 46, 31-45 (1998).

${ }^{6}$ J. C. Lin, "On microwave-induced hearing sensation," IEEE Trans. Microwave Theory Tech. MTT-25, 605-613 (1977).

${ }^{7} \mathrm{~F}$. Caspers and J. Conway, "Measurement of power density in a lossy material by means of electromagnetically-induced acoustic signals for non-invasive determination of spatial thermal absorption in connection with pulsed hyperthermia," Proceedings of the 12th European Microwave Conference, 1982, pp. 565-568.

${ }^{8}$ J. L. Su and J. C. Lin, "Thermoelastic signatures of tissue phantom absorption and thermal expansion," IEEE Trans. Biomed. Eng. 43, 178-182 (1987).

${ }^{9}$ J. S. K. Wan, “Microwaves and chemistry: the catalysis of an exciting marriage," Rev. Chem. Intermed. 19, 147-158 (1993).

${ }^{10}$ T. Bowen, L. Nasoni, A. E. Pifer, and G. H. Sembrosk, "Some experimental results on the thermoacoustic imaging of soft tissue-equivalent phantoms,"' Ultrason. Symp. Proc. 2, 823-827 (1981).

${ }^{11}$ R. G. Olsen, "Generation of acoustic images from the absorption of pulsed microwave energy," in Acoustic Imaging, edited by J. P. Powers (Plenum, New York, 1982), pp. 53-59.

${ }^{12}$ R. G. Olsen and J. C. Lin, "Acoustic imaging of a model of a human hand using pulsed microwave irradiation," Bioelectromagnetics (N.Y.) 4 397-400 (1983).

${ }^{13}$ J. C. Lin and K. H. Chan, "Microwave thermoelastic tissue imagingsystem design," IEEE Trans. Microwave Theory Tech. 32, 854-860 (1984).

${ }^{14}$ R. L. Nasoni, G. A. Evanoff, Jr., P. G. Halverson, and T. Bowen, "Thermoacoustic emission by deeply penetrating microwave radiation," Ultrason. Sym. Proc. 5, 633-637 (1984).

${ }^{15}$ K. H. Chan and J. C. Lin, "Microwave-induced thermoacoustic tissue imaging," Proceeding of the Annual International Conference of the IEEE Engineering in Medicine and Biology Society (New Orleans, Louisiana, November 4-7, 1988), pp. 445-446 (1988).

${ }^{16}$ R. A. Kruger, D. R. Reinecke, and G. A. Kruger, "Thermoacoustic computed tomography-technical considerations," Med. Phys. 26, 1832-1837 (1999).

${ }^{17}$ R. A. Kruger, K. K. Kopecky, A. M. Aisen, D. R. Reinecke, G. A. Kruger, and W. L. Kiser, Jr., "Thermoacoustic CT with radio waves: a medical imaging paradigm," Radiology 211, 275-278 (1999).

${ }^{18}$ L. V. Wang, X. Zhao, H. Sun, and G. Ku, "Microwave-induced acoustic imaging of biological tissues," Rev. Sci. Instrum. 70, 3744-3748 (1999).

${ }^{19} \mathrm{G}$. Ku and L. V. Wang, "Scanning thermoacoustic tomography in biological tissue," Med. Phys. 27, 1195-1202 (2000).

${ }^{20}$ A. A. Oraevsky, S. L. Jacques, R. O. Esenaliev, and F. K. Tittel, “Laserbased optoacoustic imaging in biological tissues," in Laser-Tissue Interaction $V$, edited by S. L. Jacques, Proceedings of the Society of Photo-Instrument Engineering, 1994, Vol. 2134A, pp. 122-128.

${ }^{21}$ R. A. Kruger and P. Liu, "Photoacoustic ultrasound: pulse production and detection of 0.5\% Liposyn," Med. Phys. 21, 1179-1184 (1994).

${ }^{22}$ A. A. Oraevsky, R. Esenaliev, F. K. Tittel, M. R. Ostermeyer, L.-H. Wang, and S. L. Jacques, "Laser opto-acoustic imaging of turbid media: determination of optical properties by comparison with diffusion theory and Monte Carlo simulation,' Laser-Tissue Interaction VII, 1996, Vol. 2681, pp. 277-284.

${ }^{23}$ C. G. A. Hoelen, F. F. M. Demul, R. Pongers, and A. Dekker, "Threedimensional photoacoustic imaging of blood vessels in tissue,' Opt. Lett. 23, 648-650 (1998).

${ }^{24}$ A. A. Oraevsky, V. A. Andreev, A. A. Karabutov, and R. O. Esenaliev, "Two-dimensional optoacoustic tomography: transducer array and image reconstruction algorithm," in Laser-Tissue Interaction X: Photochemical, Photothermal, and Photomechanical, edited by S. L. Jacques, G. J. Mueller, A. Roggan, and D. H. Sliney, Proceedings of the Society of Photo-Instrument Engineering, 1999, Vol. 3601, pp. 256-267.

${ }^{25}$ C. Gabriel, S. Gabriel, and E. Corthout, "The dielectric properties of biological tissues: I. literature survey,” Phys. Med. Biol. 41, 2231-2249 (1996).

${ }^{26} \mathrm{~S}$. Gabriel, R. W. Lau, and C. Gabriel, "The dielectric properties of biological tissues: II. measurements in the frequency range $10 \mathrm{~Hz}$ to 20 GHz,' Phys. Med. Biol. 41, 2251-2269 (1996).

${ }^{27}$ S. Gabriel, R. W. Lau, and C. Gabriel, "The dielectric properties of biological tissues: III. parametric models for the dielectric spectrum of tissues," Phys. Med. Biol. 41, 2271-2293 (1996).

${ }^{28}$ C. C. Johnson and A. W. Guy, "Nonionizing electromagnetic wave effects in biological and system,"' Proc. IEEE 60, 692-718 (1972).

${ }^{29}$ S. Chaudhary, R. Mishra, A. Swarup, and J. Thomas, "Dielectric properties of normal \& malignant human breast tissues at radiowave and microwave frequencies," Indian J. Biochem. Biophys. 21, 76-79 (1984).

${ }^{30}$ W. Joines, R. Jirtle, M. Rafal, and D. Schaeffer, "Microwave power absorption differences between normal and malignant tissue," Int. J. Radiat. Oncol., Biol., Phys. 6, 681-687 (1980).

${ }^{31}$ W. Joines, Y. Zhang, C. Li, and R. Jirtle, "The measured electrical properties of normal and malignant human tissues from 50-900 MHz," Med. Phys. 21, 547-550 (1994).

${ }^{32}$ K. R. Foster and J. L. Schepps, "Dielectric properties of tumor and normal tissues at radio through microwave frequencies," J. Microwave Power 16, 107-119 (1981). 